\title{
Hysteroscopy in Uterine Anomalies: A Boon!
}

\author{
${ }^{1}$ Charu Pathak, ${ }^{2}$ Rahul Manchanda, ${ }^{3}$ Garima Yadav
}

\begin{abstract}
Introduction: The incidence of congenital uterine malformations is as high as 3 to $4 \%$ with septate uterus being one of the most common forms of congenital malformation. Structurally anomalous uterus has been recognized as a cause of infertility, and obstetric complications. Of all known uterine anomalies, septate uterus is the most common and is associated with poorest reproductive outcome, with fetal survival rates between 6 to $28 \%$. Also, it carries high rate of spontaneous miscarriage exceeding $>60 \%$ but, on the bright side, it is one of the uterine anomaly that is most amenable via simple hysteroscopic management.
\end{abstract}

Aims and objectives: To describe a case series (comprising two cases) septate uterus managed successfully using hysteroscopy.

Background: Septate uterus results from incomplete resorption of paramesonephric mullerian ducts during the first trimester of pregnancy. Depending on the size of septum, it can be incomplete septum or complete septum dividing the uterine cavity into two separate components including two cervix and vaginal septum. Congenital malformations may be associated with recurrent pregnancy loss, preterm labor, abnormal fetal presentation, intrauterine growth restriction (IUGR) and infertility.

Hysteroscopy is considered the gold standard for the assessment and treatment of intrauterine anomalies.

Cases: Authors report two cases of septate uterus managed hysteroscopically.

- A case of complete septate uterus and another case of complete septate uterus with two cervices, managed hysteroscopically both subjects conceived successfully after treatment.

Conclusion: Operative hysteroscopy is an effective and safe minimally invasive technique to manage complete uterine septum, associated with quicker recovery. Additionally, there is no scar formation which promotes improved reproductive outcome. It has enabled more liberalized approach to treatment, i.e. now being extended to include patients with recurrent pregnancy loss and premature labor. Also, this intervention can be used successfully in patients diagnosed with infertility secondary to uterine septations, especially if in vitro fertilization (IVF) is being contemplated.

Keywords: Hysteroscopy, Minimally invasive, Septations, Uterine anomalies.

How to cite this article: Pathak C, Manchanda R, Yadav G. Hysteroscopy in Uterine Anomalies: A Boon! J South Asian Feder Obst Gynae 2016;8(1):74-76.

\footnotetext{
${ }^{1,3}$ Fellow, ${ }^{2}$ Head

${ }^{1-3}$ Department of Gyn-Endoscopy, Manchanda's Endoscopic Centre, New Delhi, India
}

Corresponding Author: Charu Pathak, Fellow, Department of Gyn-Endoscopy, Manchanda's Endoscopic Centre, New Delhi India, e-mail: drcharupathak@gmail.com
Source of support: Nil

Conflict of interest: None

Date of received: 22 November 2015

Date of acceptance: 19 February 2016

Date of publication: March 2016

\section{INTRODUCTION}

The incidence of congenital malformations is as high as 1 or 2 per 1000 women to 15 per 1000 women with septate uterus being one of the most common form of congenital malformation. ${ }^{1,2}$ Pooled data suggests that prevalence of septate uterus in women with normal fertility ${ }^{1}$ and infertile females ${ }^{3}$ is similar (approximately $1 \%$ ), although the prevalence in women with recurrent pregnancy loss is little higher $3.3 \%{ }^{1}$ Septate uterus is associated with poorest reproductive outcome, with fetal survival rates between 6 and $28 \%$ and high rate of spontaneous miscarriage exceeding $>60 \%{ }^{4}$ but, on a brighter side, it is the type of uterine anomaly most amenable to hysteroscopic treatment. Nearly, $40 \%$ patients with septate uterus have reproductive failure, obstetric complications like intrauterine growth restriction (IUGR) and preterm birth. The first hysteroscopic septum resection was performed in 1974, and the first successful outcome following this procedure was in $1981 .^{5}$

\section{CASE REPORTS}

Authors report two cases of complete uterine septum.

- Thirty-three years old female came with complaint of pain in lower abdomen with history of secondary infertility and two miscarriages, diagnosed as complete uterine septum with two cervices (bicollis). Diagnostic and operative laparoscopy and hysteroscopy was advised. Hysteroscopic septal resection was performed with resectoscope keeping both cervices intact under GA in early proliferative phase. She conceived and delivered a term healthy baby girl by lower segment cesarean section (LSCS) (McDonald's circlage done including both cervices). Intraoperatively, uterus was normal and no septum was seen.

- The second patient is 26 years old P0L0 A2 came as a case of secondary infertility. She had two spontaneous abortions at 8 and 9 weeks respectively. Hysterosalpingogram revealed bicornuate uterus with partial uterine septum, bilateral tubes were patent with free spillage (Fig. 1). Ultrasound showed 


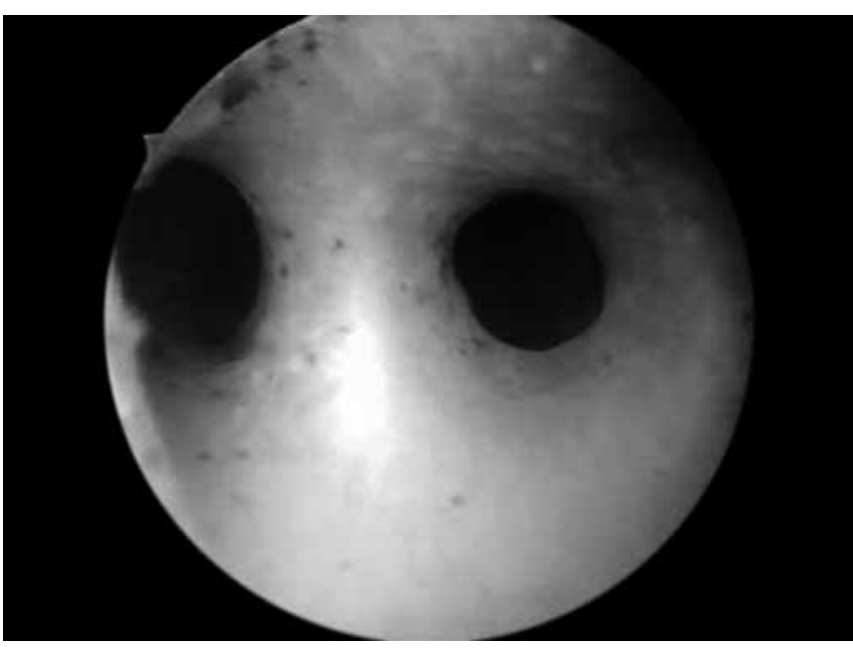

Fig. 1: Hysteroscopic view of uterine septa dividing the uterine cavity into two halves

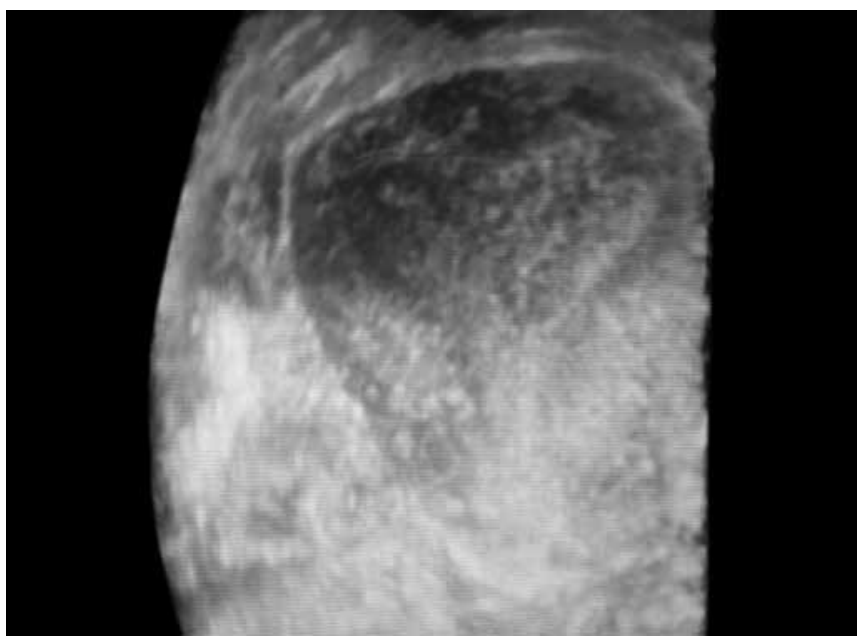

Fig. 2: Ultrasound picture of bicornuate uterus with echogenic partial midline septum and cavities on both sides

bicornuate uterus (Fig. 2), thus, a diagnostic and operative laparoscopy and hysteroscopy was advised. Hysteroscopic septoplasty was performed with scissors (Fig. 3) and intrauterine device (IUD) was inserted after removing copper coil. On laparoscopy, uterus was normal in size with broad fundus. Her relook hysteroscopy done a month later revealed few adhesions for which adhesiolysis was done and cavity was normalized (Fig. 3). She was advised for normal trial of conception. Her positive $\beta$-human chorionic gonadotropin ( $\beta$-hCG) levels confirmed pregnancy on 22 June 2015, transvaginal scan showed 6 weeks and 4 days intrauterine pregnancy.

\section{DISCUSSION}

American Fertility Society (AFS) has classified uterovaginal anomalies into four types (I to IV) with further subclassification. Septate uterus recognized as class III B. It represents the most common congenital anomaly, which occurs due to inadequate resorption of mullerian

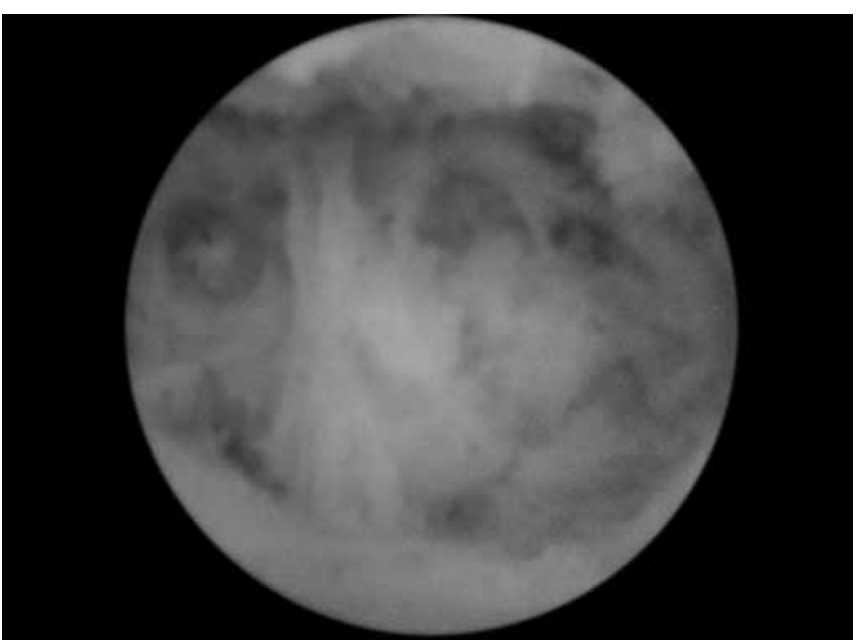

Fig. 3: Postoperative relook hysteroscopic view of uterine cavity after septum resection

ducts. ${ }^{1,2}$ It can be either complete, extending up to the cervix or partial septum (subseptate). Uterine septum is associated with obstetric complications like breech presentation, oblique and transverse lie, preterm labor, retained placenta, recurrent miscarriage and infertility. ${ }^{4}$ The two cases reported had some form of septate uterus. The first subject had complete septate uterus with two cervices (bicollis) and two uterine cavities, whereas the second subject had a complete septum with two cavities and a single cervix, having history of recurrent miscarriages followed by secondary infertility. There are different modalities to diagnose congenital uterine anomalies like hysterosalpingogram, ultrasound and magnetic resonance imaging (MRI). Magnetic resonance imaging being the most sensitive modality to diagnose septate uterus. ${ }^{1,6}$ However, combined hysteroscopy and laparoscopy is considered gold standard technique for assessing congenital uterine anomalies as it helps to differentiate septate uterus from bicornuate uterus by allowing visualization the serosal surface of uterus laparoscopically.

The primary indication of uterine septal resection is recurrent miscarriages or fetal loss. By restoring the normal anatomy of the uterine cavity, septoplasty enhances the chances of successful embryo implantation and, therefore, a favorable obstetric outcome. The introduction of hysteroscopy to diagnose and treat intrauterine conditions in general and septate uterus in particular supplants the traditional method performed via laparotomy approach (abdominal metroplasty). Hysteroscopy has revolutionized the uterine morbidity management globally. Its advantages over traditional abdominal approach include less morbidity, less postprocedure pain, reduced hospital stay, thereby making it a cost-effective procedure. Given its minimally invasive approach, there is no scar formation (a genuine cosmetic 
concern, especially in unmarried girls) or postoperative adhesions, which allows the maintenance of integrity of uterine wall. The recovery time before conception is shortened. Also, the probability of profuse bleeding and trauma are decreased when compared to abdominal approach. All these advantages make hysteroscopy a better option.

There are different methods of performing operative hysteroscopy. It may be performed using monopolar, bipolar electrocautery, argon laser or scissors, none of the particular modality being superior to other. ${ }^{4,9}$ After hysteroscopic septoplasty, pregnancy rates and live birth rates are 67 and $57.5 \%$ respectively. ${ }^{7}$ The two cases discussed are operated by two different modalities hysteroscopically, the first case was performed 11 years ago in which resectoscope was used for septal resection. The second case performed in 2015 in which scissors were used for hysteroscopic septal resection. Fortunately, after the procedure both patients conceived spontaneously. The first subject delivered a live healthy girl by LSCS. The second subject conceived within 3 months of procedure doing well. This early conception is unusual given that the most women conceived between 8 and 14 months after hysteroscopic metroplasty ${ }^{8}$ and has not been reported in medical literature to best of our knowledge. Both hysteroscopic modalities provided a favorable outcome, which support the argument that the type of method does not determine the obstetrical success rate. Innovations in instrumentation and safe energy sources will help in continuous improvement of safety and simplicity of this technique.

\section{CONCLUSION}

Hysteroscopy offers a minimally invasive alternative to traditional with metroplasty. This abdominal technique is associated less morbidity, minimal postoperative pain, no scarring, less surgical adhesions, shorter hospital stay, lower cost, and expedited recovery provide an unprecedented obstetric outcome leading to its widespread global acceptance. Over last four decades, the technique has now become the face of metroplasties done worldwide with minimal change in the original technique described.

\section{REFERENCES}

1. Valle RF, Ekpo GE. Hysteroscopic metroplasty for the septate uterus: review and meta-analysis. J Minim Invasive Gynecol 2013 Jan-Feb;20(1):22-42.

2. Acien $P$, Acien M. Evidence-based management of recurrent miscarriage. Surgical management. Int Congr Series 2004; 1(266):342-355.

3. Mollo A, De Franciscis P, Colacurci N, Cobellis L, Perino A, Venezia R, Alviggi C, De Placido G. Hysteroscopic resection of the septum improves the pregnancy rate of women with unexplained infertility: a prospective controlled trial. Fertility Sterility 2009 June;91(6):2628-2631.

4. Heinonen PK, Saarikoski S, Pystynen P. Reproductive performance of women with uterine anomalies. An evaluation of 182 cases. Acta Obstet Gynecol Scand 1982;61(2):157-162.

5. Chervenak FA, Neuwirth RS. Hysteroscopic resection of the uterine septum. Am J Obstet Gynecol 1981 Oct 1;141(3):351-343.

6. Pellerito JS, Mc carthy SM, Doyle MB, et al. Diagnosis of uterine anomalies: relative accuracy of MR imaging, endovaginal sonography and hysterosalpingography. Radiol 1992;183(3):795-800.

7. Esmaeilzadeh S, Delavar MA, Andarieh MG. Reproductive outcome following hysteroscopic treatment of uterine septum. Mater Sociomed 2014 Dec;26(6):366-371.

8. Pabuccu R, Gormel V. Reproductive outcome after hysteroscopic metroplasty in women with septate uterus and otherwise unexplained infertility. Fertiliy Sterility 2004;(6): 224-226.

9. Colacurci N, De Placido G, Mollo A, et al. Reproductive outcome after hysteroscopic metroplasty. Europ J Obstet Gynecol Reproduct Biol 1966;66:147-150. 